Kansas State University Libraries

New Prairie Press

\title{
ASSESSING REFINEMENTS IN MODELING SINUSOIDAL CONDITIONS USED TO DRIVE CATTLE BODY TEMPERATURES
}

\author{
Sheng Feng \\ Anne Parkhurst \\ Kent Eskridge \\ Daryl Travnicek \\ Donald Spiers
}

See next page for additional authors

Follow this and additional works at: https://newprairiepress.org/agstatconference

Part of the Agriculture Commons, and the Applied Statistics Commons

\section{(c)}

This work is licensed under a Creative Commons Attribution-Noncommercial-No Derivative Works 4.0 License.

\section{Recommended Citation}

Feng, Sheng; Parkhurst, Anne; Eskridge, Kent; Travnicek, Daryl; Spiers, Donald; Hahn, G. L.; and Mader, Terry (2001). "ASSESSING REFINEMENTS IN MODELING SINUSOIDAL CONDITIONS USED TO DRIVE CATTLE BODY TEMPERATURES," Conference on Applied Statistics in Agriculture. https://doi.org/10.4148/ 2475-7772.1230

This is brought to you for free and open access by the Conferences at New Prairie Press. It has been accepted for inclusion in Conference on Applied Statistics in Agriculture by an authorized administrator of New Prairie Press. For more information, please contact cads@k-state.edu. 
Author Information

Sheng Feng, Anne Parkhurst, Kent Eskridge, Daryl Travnicek, Donald Spiers, G. L. Hahn, and Terry Mader 


\title{
ASSESSING REFINEMENTS IN MODELING SINUSOIDAL CONDITIONS USED TO DRIVE CATTLE BODY TEMPERATURES
}

\author{
Sheng Feng ${ }^{1}$, Anne Parkhurst ${ }^{1}$, Kent Eskridge ${ }^{1}$, Daryl Travnicek ${ }^{1}$, \\ Donald Spiers ${ }^{2}$, G. L. Hahn ${ }^{3}$, and Terry Mader ${ }^{4}$
}

1. Department of Biometry, University of Nebraska

2. Department of Animal Science, University of Missouri - Columbia

3. U.S. Meat Animal Research Center, U.S.D.A, Clay Center, NE

4. Department of Animal Science, University of Nebraska

\begin{abstract}
A model, termed the PET model, is used to estimate body temperature in cattle challenged by hot cyclic chamber temperatures. The model is based on Newton's law of cooling, driven by an estimated sinusoidal function. In practice, it is often difficult to maintain hot sinusoidal fluctuations in chamber temperatures. However, it is possible to model cyclic chamber temperatures using a discrete Fourier series. By increasing the precision in estimating the cyclic temperature driving function, we can more precisely estimate the parameters in the PET model. Simulation studies were performed to investigate the effect of under- and over-parameterization on accuracy of estimates, performance of a number of model selection criteria, and on nonlinear behavior such as intrinsic and parameter-effects curvature, bias, excess variance, and skewness. Our results will help researchers decide how to model ambient temperatures producing heat stress in cattle and improve estimates for evaluating management strategies.
\end{abstract}

\section{Introduction}

The performance, health, and well-being of cattle are strongly influenced by climate. Managing cattle to reduce the impact of hot weather remains a challenge. There is a need for further development of an environmental management system to guide feeder cattle managers in making strategic and tactical decisions prior to and during hot weather. To enhance our understanding of cattle response to high temperature, we use statistical models to describe their responses (i.e. body temperature change) to weather changes and quantitatively characterize an animal's state of well-being.

Researchers have pointed out that, under heat stress, the cattle body temperature $\left(\mathrm{T}_{\mathrm{B}}\right)$ changes cyclically following the air temperatures (Ta) [1] and $\mathrm{Ta}$ is thought to be the principal driving force for $T_{B}$. To estimate $T_{B}$, we assume that Ta affects $T_{B}$ following 'Newton's law of cooling": the rate of change in animal body temperature, from the body core to its surface, is proportional to the difference in temperature between the body core and its environment, or written in a one-parameter mathematical model:

$$
\frac{\partial T_{B}}{\partial t}=K\left(T a-T_{B}\right) \text {. }
$$


Thus, $T_{B}$ can be solved as:

$$
T_{B}=K e^{-K t} \int_{0}^{t} e^{K t} T_{a} d t+T_{\text {в } 0} \cdot e^{-K t} \text { where } T_{B 0}=\text { initial } T_{B}
$$

In this expression, $\mathrm{T}_{\mathrm{B}}$ is determined by the ambient temperature Ta and time $t$. Thus modeling Ta is a critical first step to estimate $T_{B}$.

Predicting naturally varying environmental air temperature is difficult. However, for our experiment, where animals are housed in controlled cyclic (i.e. sinusoidal, $33 \pm 7^{\circ} \mathrm{C}$,) conditions, Ta may be easily estimated by a sinusoidal function.

The PET (Parkhurst, Eskridge and Travnivek) model has been built based on this assumption [2]. In this model, $\mathrm{Ta}$ is fitted first with a sinusoidal function, then $\mathrm{T}_{\mathrm{B}}$ is predicted by solving equation $I$.

However, it would be very useful to find a model for the relationship between $T_{B}$ and Ta that does not require knowledge of the specific form for $\mathrm{Ta}$. In practice, it is often difficult to maintain hot sinusoidal fluctuations in chamber temperatures. This causes problems in predicting $T_{B}$. Fourier's series can be used to address this issue. The question is, whether the Fourier series brings significant improvement in $\mathrm{T}_{\mathrm{B}}$ prediction.

In this study, the PET model is extended by modeling Ta with a Fourier series. This gives a series of Ta models with a different number of parameters (i.e., with one more "period", there will be two more parameters in the model - refer to materials and methods). In order to know how many periods are needed for the Fourier series to adequately model Ta, several model selection criteria are investigated. However, the selected model is only known to be favored by the model selection criteria, it is not known whether it performs better than other candidate models. Thus, it is necessary to compare the selected model with under- and over-parameterized (less and more periods) models.

Simulation studies are conducted to evaluate the importance of using a Fourier series on modeling $\mathrm{T}_{\mathrm{B}}$ and address questions including model selection and model comparison.

\section{Methods}

\section{Ta and $\mathbf{T}_{\mathbf{B}}$ modeling}

(1) Model Ta with a sinusoidal function (PET model).

First, Ta is modeled by a sinusoidal function:

$$
\mathrm{T} a=\mu+\mathrm{a} \sin \lfloor\omega(\mathrm{t}-\varphi)\rfloor
$$

II.

Then, this Ta function is substituted into equation $\mathrm{I}$. With three more parameters, $\Delta, \gamma$ and $\mathrm{T}_{\mathrm{B} 0 \text {, }}$ and then $T_{B}$ is solved for as:

$$
T_{B}=e^{K t}\left[T_{B 0}+K\left(S_{0}+S_{1}\right)\right]
$$


where,

$$
\mathrm{S}_{0}=\left(\gamma \mu_{\mathrm{a}}+\Delta\right)\left(\mathrm{e}^{\mathrm{kt}} \quad 1\right) / \mathrm{K}
$$

and

$$
\mathrm{S}_{1}=\gamma \frac{\alpha}{\sqrt{\mathrm{K}^{2}+\omega^{2}}}\left[\mathrm{e}^{\mathrm{kt}} \sin (\omega(\mathrm{t} \quad \varphi) \quad \theta)+\sin (\omega \varphi+\theta)\right] .
$$

The three additional parameters in the model are defined as:

a scaling factor $\gamma$, which can be thought of as the proportion of variance in $T_{B}$ comparable to variance in Ta; $\Delta=\overline{\mathrm{T}}_{\mathrm{B}}-\gamma \overline{\mathrm{T}} \mathrm{a}$ where $\Delta$ is the gradient between mean $\mathrm{T}_{\mathrm{B}}$ and mean Ta adjusted for $\gamma$; and $\mathrm{T}_{\mathrm{B} 0}$, the steer body temperature at $\mathrm{t}=0$, is the starting value set for the PET model.

Under ideal conditions, when Ta is a well-controlled sinusoidal function, this method results in good $T_{B}$ estimation. However, practically it is very difficult to maintain precise sinusoidal fluctuations in chamber temperatures, so the PET model needs to be improved to be more useful in practice. This improved model, which we now call the extended PET model, does not depend on a specific analytic function of $\mathrm{Ta}$, and may provide a basis for extending the application to real weather heat challenge.

(2) Extended PET model: Fourier series.

Next, Ta is modeled as:

$$
T_{a}=\mu_{a}+\sum_{i=1}^{n} a_{i} \sin \left[i \omega\left(t-\phi_{i}\right)\right]
$$

This is an alternative form of the classic expression of Fourier series. Consequently, $T_{B}$ can be solved as:

$$
\begin{array}{r}
T_{B}=e^{-K t}\left[T_{B 0}+K\left(S_{0}+S\right)\right] \\
S_{0}=\left(\gamma \mu_{a}+\Delta\right)\left(e^{k t}-1\right) / K
\end{array}
$$

where

$$
S=\sum S_{i}=\gamma \sum \frac{\alpha_{i}}{\sqrt{K^{2}+(i \omega)^{2}}}\left[e^{k t} \sin \left(i \omega\left(t-\phi_{i}\right)-\theta_{i}\right)+\sin \left(i \omega \phi_{i}+\theta_{i}\right)\right]
$$


and

$$
\sin \theta_{\mathrm{i}}=\frac{\mathrm{i} \omega}{\sqrt{(\mathrm{i} \omega)^{2}+\mathrm{K}^{2}}} \quad \cos \theta_{i}=\frac{K}{\sqrt{(i \omega)^{2}+K^{2}}}
$$

\section{Model Selection}

Since a discrete Fourier series is used to model the Ta curve, we need to know how many period terms are adequate for the "best model" and find a "stop-criterion". Introduction of more periods into the model improves the fit for Ta; however, for this data set, when more than three periods are used in fitting $\mathrm{Ta}$, there is no obvious improvement in estimating $\mathrm{T}_{\mathrm{B}}$. More generally, in order to choose the best model from the set of candidate models, we need to know when to stop adding periods to the Fourier series in fitting Ta. Model selection criteria that are evaluated include:

(1) Mean Square Error (MSE), (2) Information criteria and (3) Nonlinear behavior

For the information criteria, $\mathrm{n}$ is the number of observations and $\mathrm{m}$ is the total number of free parameters in the model.

A. Akaike Information Criterion [3]

$$
A I C=n\left(\log \hat{\sigma}^{2}+1\right)+2 m
$$

B. Akaike Information Criterion C (AICc) [4]

$$
\begin{aligned}
A I C c & =n \log \hat{\sigma}^{2}+n(m+n) /(n-m-2) \\
& =A I C+2(m+1)(m+2) /(n-m-2)
\end{aligned}
$$

C. Consistent AIC (CAIC) [6]

$$
C A I C=n\left(\log \hat{\sigma}^{2}\right)+m[(\log n)+1]
$$

D. Schwarz Bayesian Criterion (SBC) [7]

$$
S B C=n\left(\log \hat{\sigma}^{2}\right)+m \log n
$$

E. Hannan and Quinn Criterion (HQ) [8]

$$
H Q=n\left(\log \hat{\sigma}^{2}\right)+2 m \log (\log n)
$$


Since linear approximation is used as the calculation method in the nonlinear regression, we need to check the nonlinear behavior for each of the candidate models. If the linear approximation is poor, the parameter estimates may be unreliable.

On the other hand, we may also consider those "nonlinear behavior" statistics as criteria for model selection - a "better" candidate model would have "less serious" or "more acceptable" nonlinear behavior than its competitors (this hypothesis also needs to be tested by simulation studies).

The nonlinear behavior criteria include:

A. Curvature Index

Bates and Watts [9] suggest that if the curvatures, both intrinsic and parameter-effects curvature, are large (i.e., $>0.4$ ), then the linear approximation may not be reliable.

B. Box's Bias [10]

In nonlinear regression, the discrepancy between the true value of the parameter and its estimate are generated from two sources - the model and the nonlinear procedure. Box's bias measures the latter one. The smaller the measure of Box's bias, the less serious nonlinear behaviors the model has. In the study, the percent bias is used, which expresses bias as the percentage of the true parameter value (the desired value is 0 ).

C. Relative Excess Variance [11]

In nonlinear regression, the estimated variance of parameters may be divided into two parts: one from the model (called asymptotic variance); the other from the nonlinear procedure (called excess variance). Relative excess variance measures the proportion of excess variance to asymptotic variance. A small value of relative excess variance is preferred (the desired value should be less than 1).

D. Skewness [12]

This statistic checks if the parameters are symmetrically distributed. A small value is preferred; zero skewness indicates a symmetric distribution.

\section{Simulation Studies}

The purpose of the simulation studies is to identify the "best" model selection criteria for the extended PET models and to compare different period models to evaluate the under- and overparameterization effects. We want to know whether the Fourier series extension is really an important improvement to model $\mathrm{T}_{\mathrm{B}}$.

The procedure for the simulation study is:

(1) Simulation Design: Ta is simulated under four different conditions: (A) two structures 2 and 3 periods; (B) two levels of variances for each structure - large variance 0.8 and small variance $0.005 . T_{B}$ then is obtained by solving the extended PET model equation, with two levels of variance - large variance 0.05 and small variance 0.005 . A total of 8 situations ( $2 \mathrm{Ta}$ structures by 4 variance conditions) were investigated. For each situation, 500 sets of data (Ta $\mathrm{T}_{\mathrm{B}}$ ) were generated;

(2) Select the "best" model selection criteria: Four extended PET models (with 1, 2, 3 or 4 periods in the Ta structure) were used to fit each set of generated data. Model selection criteria were calculated for each set of data and evaluated by the percentage of times they successfully selected the "correct" model. 
(3) Model comparison: the under- and over-parameterization effects were investigated by checking the distribution of each parameter (in the $T_{B}$ model) estimated from different period models; examining bias, excess-variance and skewness for each parameter; comparing the curvature and the relative efficiency between models.

\section{Results and Discussion}

\section{Select the "best" model selection criteria.}

In this study, two questions are of interest:

A. Which type of criteria (MSE, information criteria or nonlinear behavior measurements) is more likely to select the "correct" model;

B. Within the "best" type criteria, which one or ones are better able to select the correct model ( $>95 \%$ of the time);

MSE is not a good criterion for model selection. When the designed period is two, the frequency for MSE to select the true ( 2 period) model is only about $54 \%$. Though this conclusion is from simulation studies under condition 1 (high Ta variance and low $\mathrm{T}_{\mathrm{B}}$ variance), this pattern holds for all other conditions.

Our results show that the information criteria (Figure 1) perform well over a wide $\mathrm{Ta}-\mathrm{T}_{\mathrm{B}}$ range, while the nonlinear behavior measurements (Figure 2) do not work well when the variation of $T_{B}$ is relatively large (selecting the over-parameterized model). This result indicates that the information criteria are a better criteria for selecting the correct model.

Figure 2 also indicates that for the two Ta structures we investigated, the AIC criteria (AIC, AICc and CAIC) select the correct model over $95 \%$ of the time, regardless of the Ta structure and variance of $\mathrm{Ta}$ and $\mathrm{T}_{\mathrm{B}}$. The performance of $\mathrm{SBC}$ is conditional on Ta structure - when Ta is generated with two periods, SBC works well; when the Ta model is more complex (3 periods in structure), the chance for SBC to select the correct model drops lower than $95 \%$. In all cases, HQ does not work well $(<95 \%)$ - both SBC and HQ are not good criteria for our study. It can be shown that the penalty parts of the five information criteria are different. The AICc has the highest penalty while HQ has the lowest. In our simulated data, it seems that (Figure 1) the criteria with large penalty (i.e. AIC, AICc) select the true model more frequently than the criteria with small penalty (i.e. HQ).

\section{Model comparison.}

Once a Ta model has been chosen by model selection criteria, we are interested in knowing whether the selected model really has advantages over other candidate models. We compare the four candidate models, including the selected one, by investigating the curvature and relative efficiency of each model. Also, we check the distribution, the bias, the excess variance and the skewness of the same parameters in different models, exploring the effects of under- and overparameterization.

A. Criteria evaluating the model:

\section{(A.1) Curvature}

Intrinsic curvature (Figure 3) and parameter effects curvature (Figure 4) both show that the under-parameterized models have serious curvatures. However, no obvious difference can be 
detected between the selected model and the over-parameterized models. For true period $=2$, the 1 period model is the under-parameterized model, 3 and 4 period model are the overparameterized models. For true period $=3$, the 1 and 2 period models are the underparameterized model, 4 period model is the over-parameterized model.

(A.2) Relative Efficiency

We evaluate the models by applying the idea of relative efficiency. The estimated variance of each model with a different period is compared to the designed variance of the true model. It is expected that, the closer to truth the candidate model is, the closer to 1 its relative efficiency will be. Figures 5 and 6 show that the under-parameterized models have relative efficiency much larger than 1, while the over-parameterized models and the selected models have relative efficiency very close to 1 . However, there is a slight tendency for the relative efficiency of the over-parameterized models to depart from 1 as the number of periods in the model increases (the mean of the relative efficiency increases from the true model to the over-parameterized models).

B. Parameter estimation.

(B.1) Parameter distribution and bias for estimates

Figures 7 and 8 show the distribution of the parameter $K$ and its Box's bias for the estimates, respectively. The results are obtained under condition 1 , but this pattern is similar for all other conditions (Table 2). Note that the estimates of the parameters are biased when the underparameterized model is used. Over-parameterized models do not give more accurate estimates than do the selected model. They appear to be more strongly biased than the selected model, though the difference is small and hard to detect.

(B.2) Relative excess Variance and Skewness

Figures 9 and 10 show relative excess variance and skewness for the estimates of the parameter $K$, respectively.

It is obvious that the parameter estimates obtained from the under-parameterized models are not reliable - the relative excess variance is way beyond the acceptable value of 1 and the skewness measurement is poor.

Though not obvious, there is some evidence that shows the selected model is also a little "better" than the over-parameterized models. The mean of the relative excess variance and the skewness of the parameter estimates from the selected model are closer to the preferred value than those from the over-parameterized models. This indicates that, with more parameters, the over-parameterized models are not able to give more accurate and precise estimates for the parameters than does the selected model. This conclusion holds true for all other conditions.

\section{Summary}

The extended PET model derived from this study is a necessary and useful extension of the PET model (which is basically the 1 period model in the Fourier series), and leads to more precise and less biased estimation of $\mathrm{T}_{\mathrm{B}}$. Furthermore, this model does not require any prior information on any specific structure in Ta. Theoretically, any Ta curve can be modeled by a Fourier series. This is very useful when analyzing field data, where Ta has more complicated structure than chamber data;

In this study, AIC, AICc and CAIC are preferred for model selection purposes. They have been shown to select the true model more frequently than other candidate criteria (i.e. MSE, the 
nonlinear behavior statistics and other two information criteria, SBC and HQ). The power (probability of selecting the correct model) is greater than $95 \%$ for all conditions.

The under-parameterized models are much worse than the selected model. The conclusion is based on comparisons of model curvatures, model relative efficiency and parameter estimates. Over-parameterization does not seem to hurt $T_{B}$ prediction seriously, though some evidence shows that the over-parameterized model does produce a little more bias, higher excess variance, heavier skewness, larger relative efficiency and more serious curvatures than the true model. At the very least, the over-parameterized models do not produce better predictions of $T_{B}$.

The following procedure is recommended for predicting $T_{B}$ given the heat stress condition Ta: The first step is to fit Ta by applying a Fourier series, with different periods. Then, select the "best" model using model selection criteria AICc. Finally, obtain the Ta parameter estimates and solve the extended PET model equation to obtain predicted $T_{B}$.

\section{References}

[1] Hahn, G.L. 1989. Body temperature rhythms in farm animals. Proceedings of $11^{\text {th }}$ ISBCongress. SPB Academic Publishing bv, The Hague, The Netherlands. 325-337;

[2] Parkhurst, A.M., Eskridge, K.M., and Travnicek, D.A. 2000. Evaluating nonlinear mixed model methods for fitting Newton's law of cooling with a sinusoidal forcing function. (In preparation);

[3] Akaike, H. 1974. A new look at the statistical model identification. IEEE Trans. Auto. Control 19 716-723.

[4] Hurvich, C.M. and Tsai, C.L. 1989. Regression and time series model selection in small samples. Biometrika 76, 297-307.

[5] Shibata, R. 1976. Selection of the order of an autoregressive model by Akaike's information criterion. Biometrika 63, 117-126.

[6] Bozdogan, H. and Ramirez, D.E. 1988. Model selection algorithm for the orthogonal factor model using AIC and CAIC. Psychometrika 53, 407-415.

[7] Schwarz, G. 1978. Estimating the dimension of a model. The Annals of Statistics. Vol.6, No.2, 461-464.

[8] Hannan, E. and Quinn, B. 1979. The determination of the order of an autogression. J. R. Statist. Soc. Ser. B 41:190-195.

[9] Bates, D.M., and Watts, D.L. 1980. Relative curvature measures of nonlinearity. J. R. Statist. Soc. Ser. B 42:1-25.

[10] Box, M.J. 1972. Bias in nonlinear estimation. J. R. Statist. Soc. Ser. B 33:171-201. 
[11] Lowry, R. and R.Morton, 1983. An asymmetry measure for estimators in non-linear regression models. Proc. $44^{\text {th }}$ Session Int. Statist. Inst., Madrid, Contributed Papers 1:351-354.

[12] Hougaard, P., 1985. The appropriateness of the asymptotic distribution in a nonlinear regression model. J.R.Statist. Soc.Ser. B 47:103-114. 

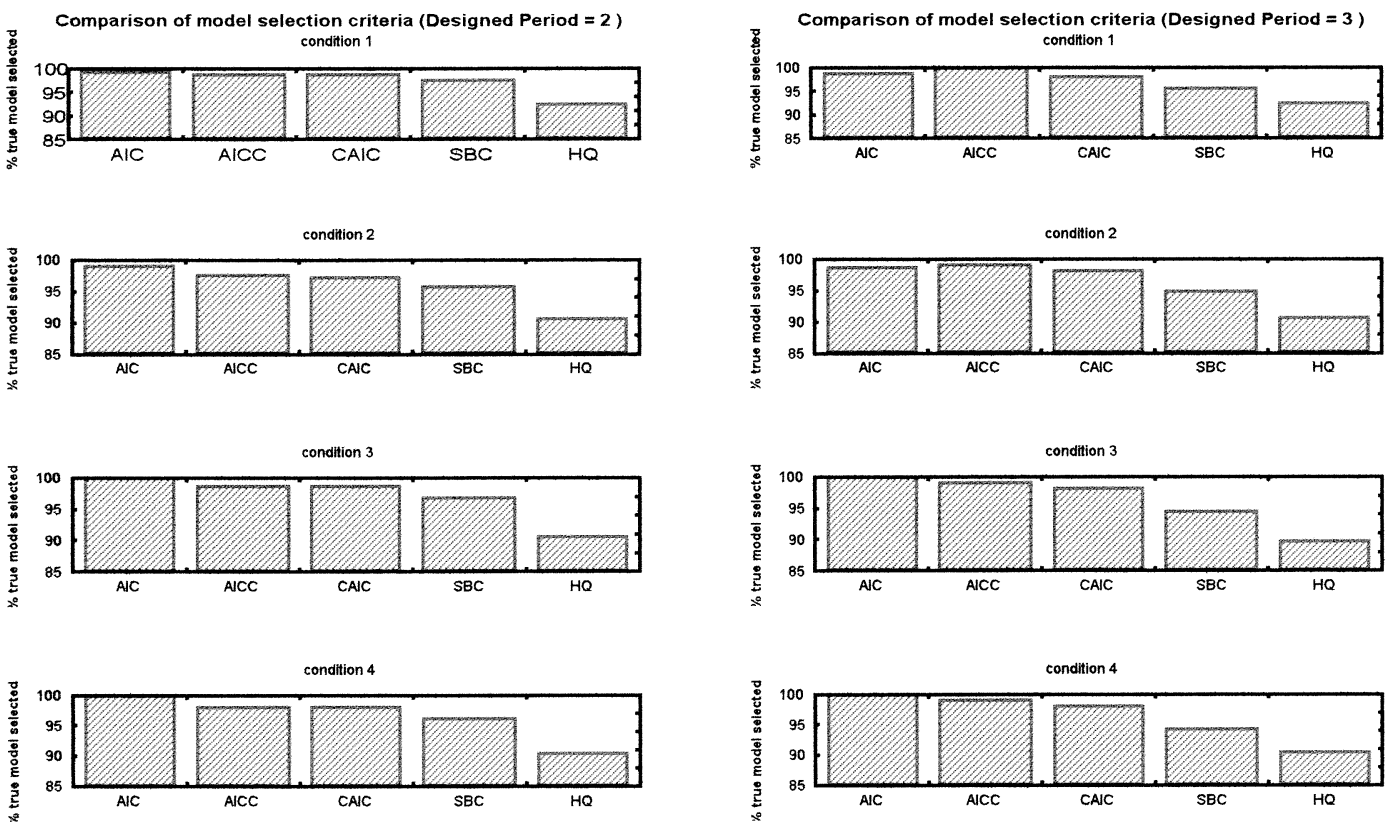

Figure 1. Simulation study on Information Criteria

All information criteria perform well, except HQ. AIC and its derivatives are the best criteria (for all conditions, the chance of selecting correct model $>95 \%$ ). Condition 1: high $\mathrm{Ta}$ variance and low $T_{B}$ variance; Condition 2 : high $T a$ variance and high $T_{B}$ variance; Condition 3: low Ta variance and low $T_{B}$ variance; Condition 4: low Ta variance and high $T_{B}$ variance.
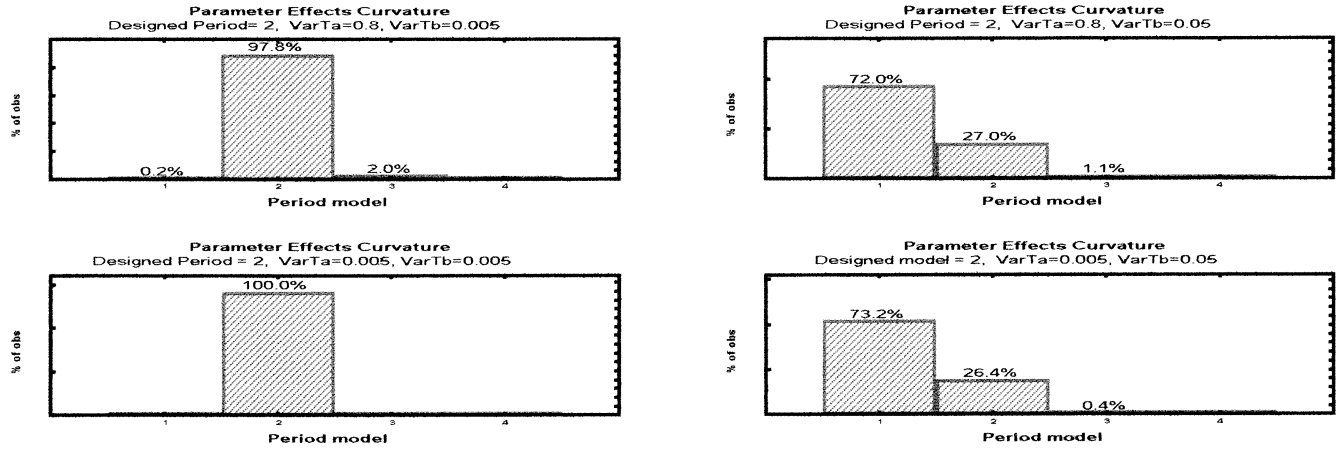

Figure 2. Simulation study on Parameter Effects Curvature.

When variance of $T_{B}$ is small ( $\operatorname{Var} T_{B}=0.005$, left column), this criteria selects the corrected model above $95 \%$ of the time; when variance of $T_{B}$ is large $\left(\operatorname{Var} T_{B}=0.05\right.$, right column), this criteria does not perform well. Other nonlinear behavior criteria share the same pattern. 

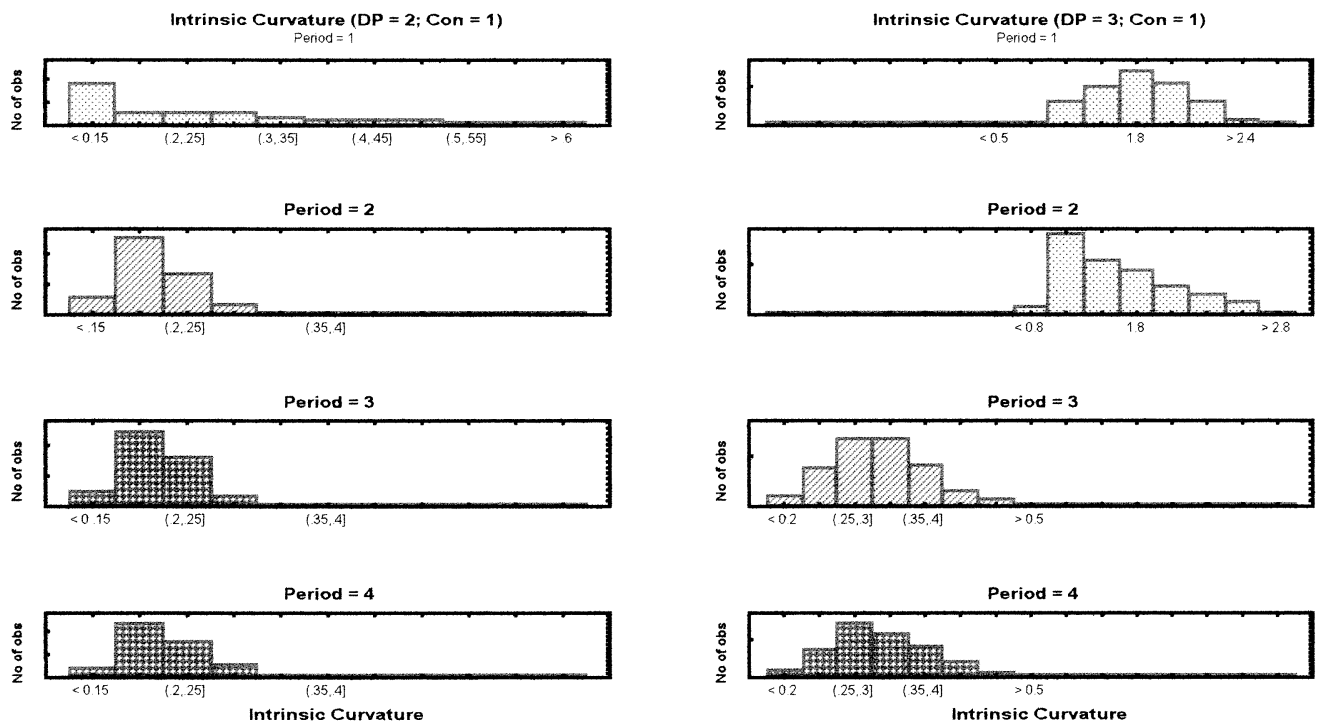

Figure 3. Intrinsic Curvature of different period model for "true" period $=2$ (left column) and "true" period $=3$ (right column).
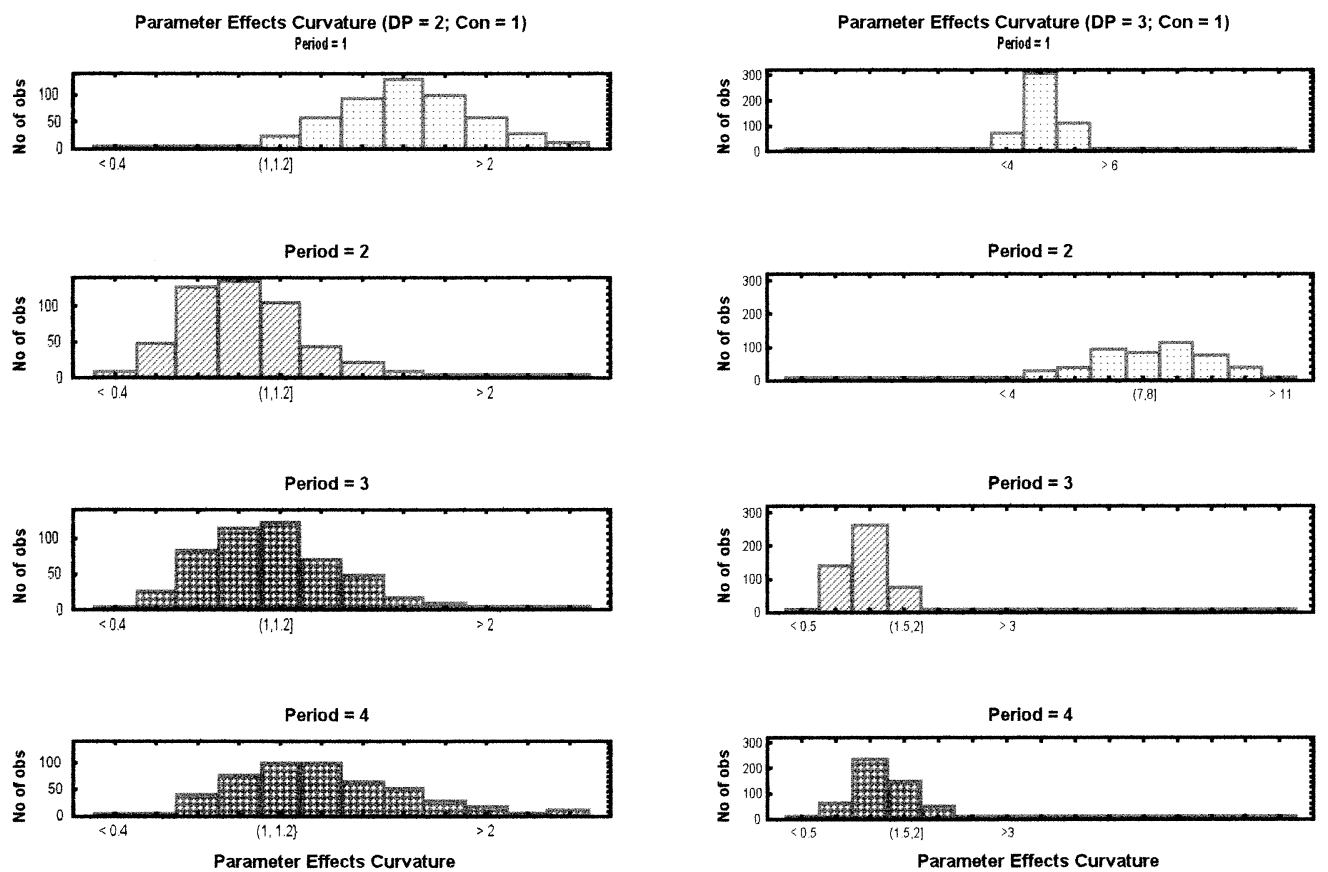

Figure 4. Parameter Effects Curvature of different period model for "true" period $=2$ (left column) and "true" period $=3$ (right column). 

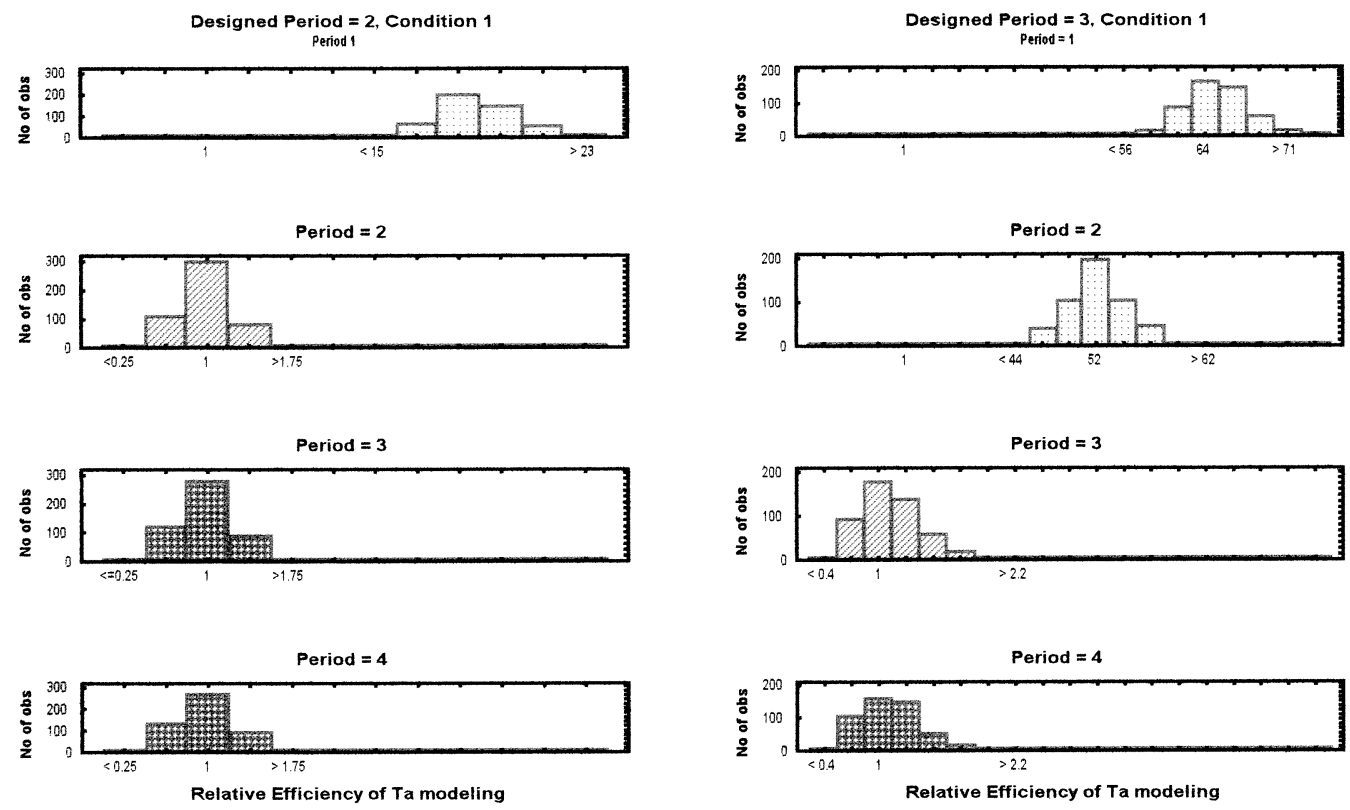

Figure 5. Relative efficiency for Ta modeling (condition 1). The relative efficiencies obtained under other conditions share this pattern.
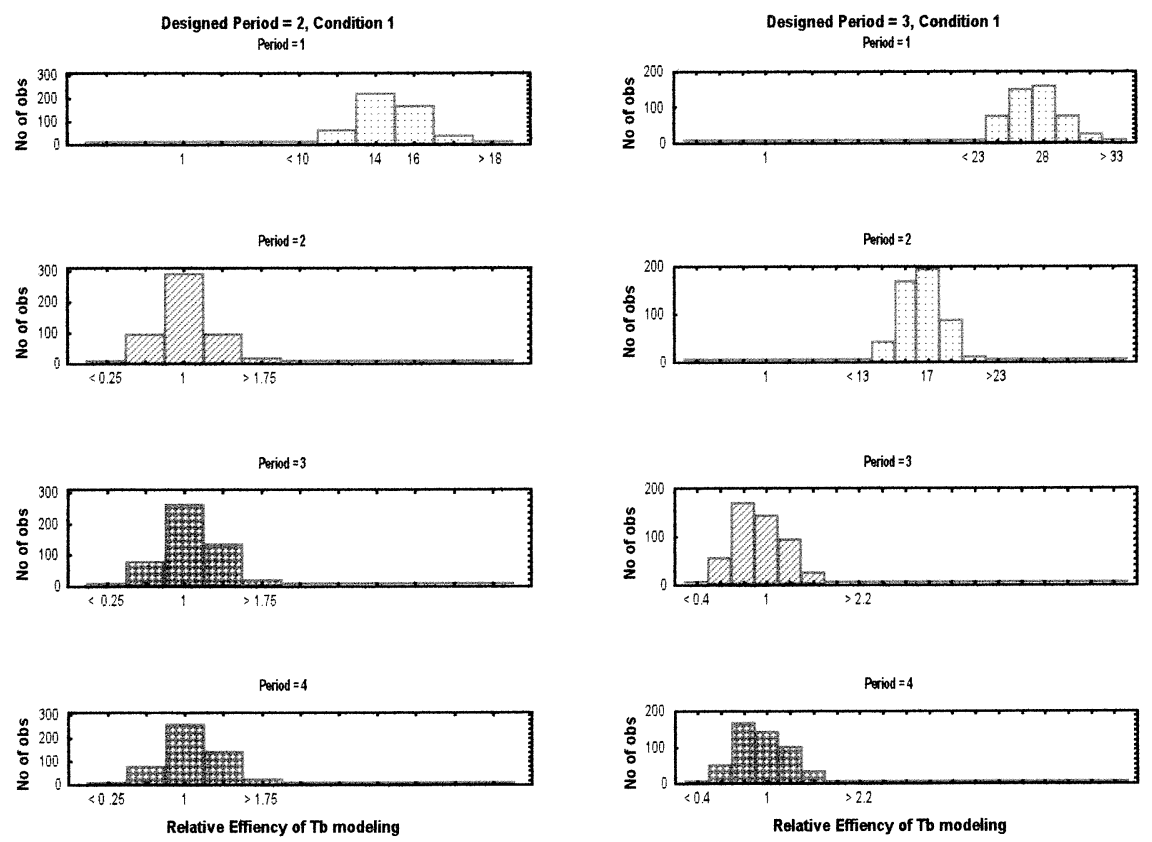

Figure 6. Relative Efficiency for $\mathrm{T}_{\mathrm{B}}$ modeling (Condition 1). The relative efficiencies obtained under other conditions share this pattern. 

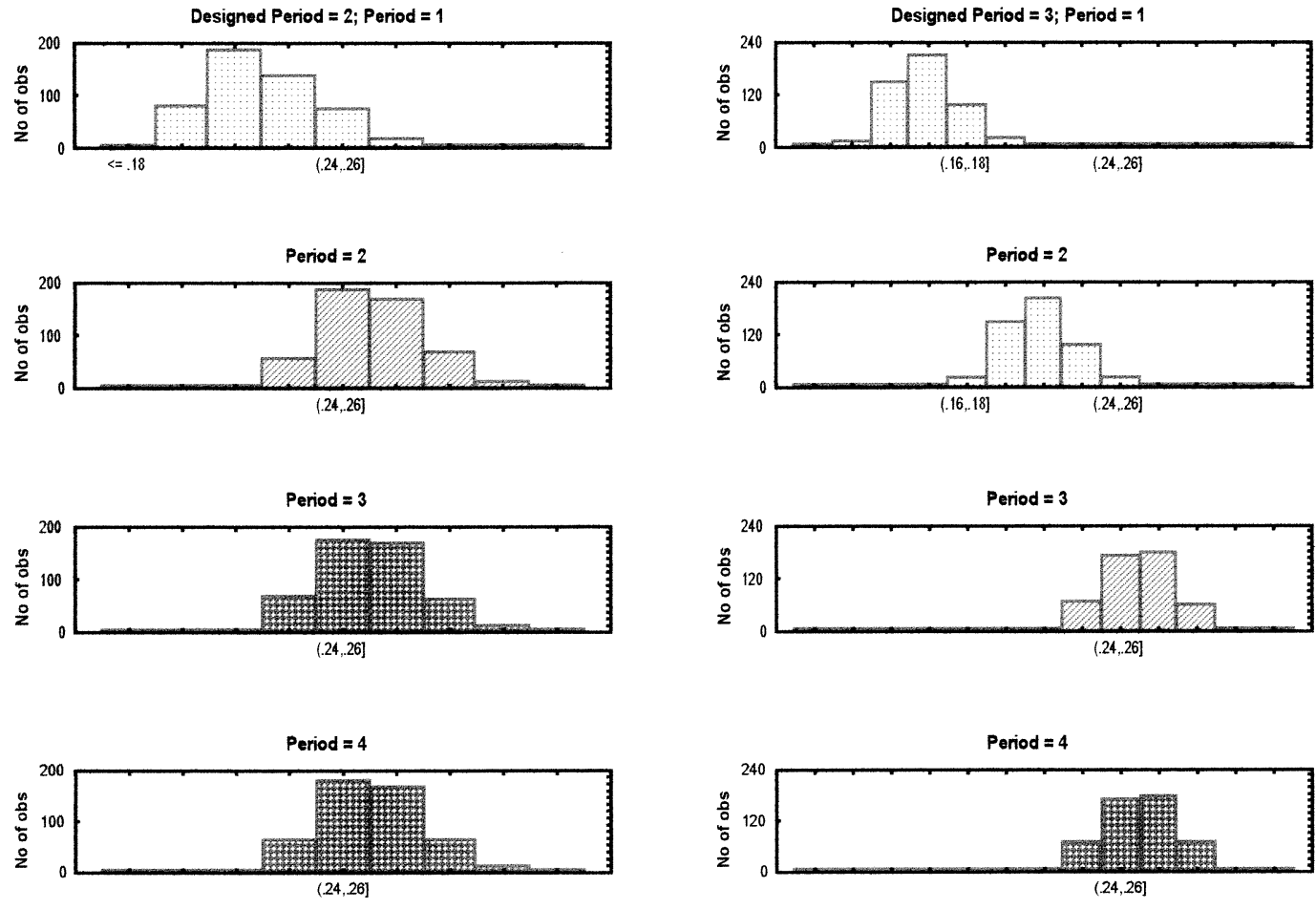

Figure 7. The distribution of $K$ (under condition 1).
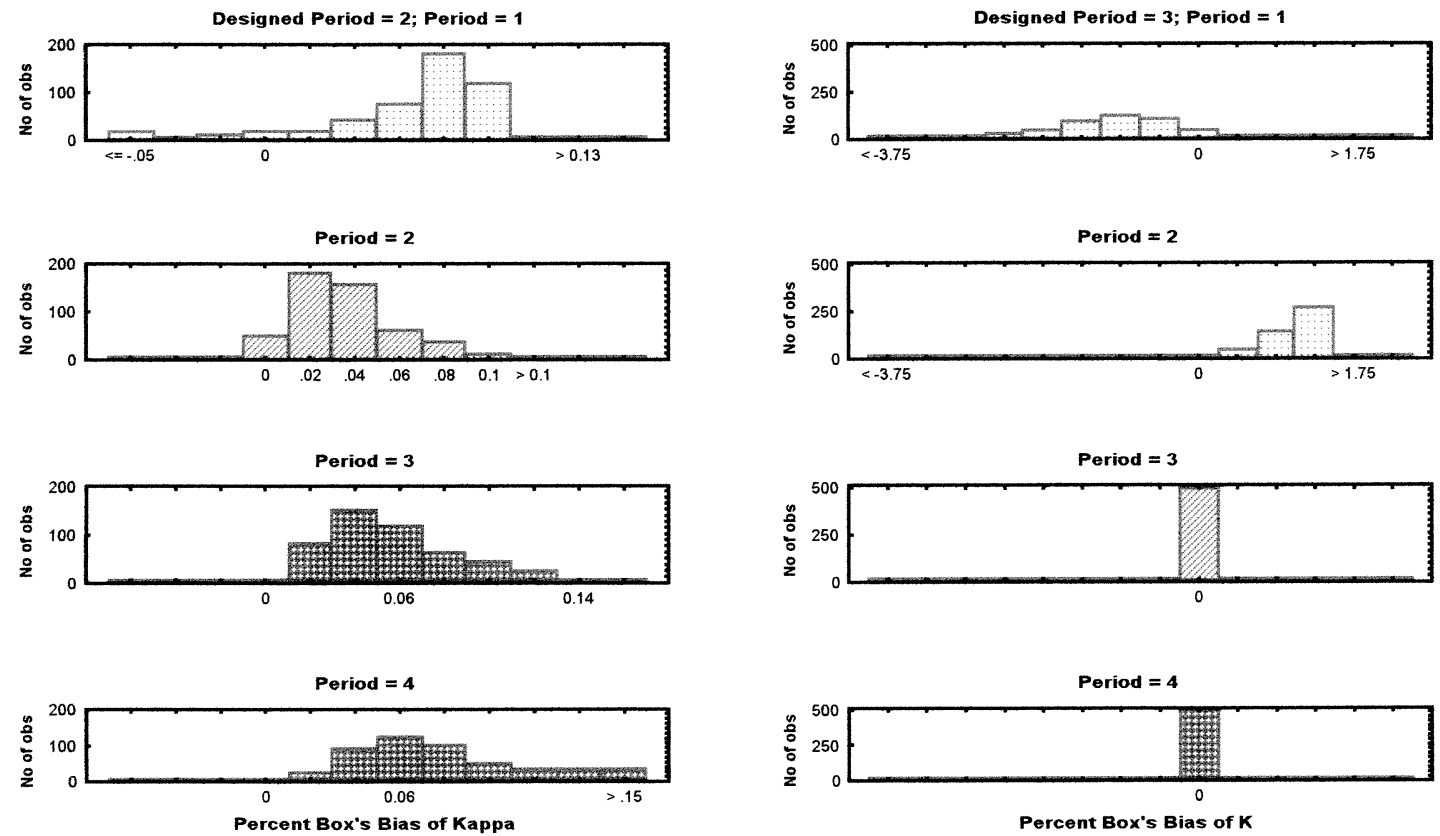

Figure 8. The percent Box's bias of the estimates of $K$ (under condition 1). 

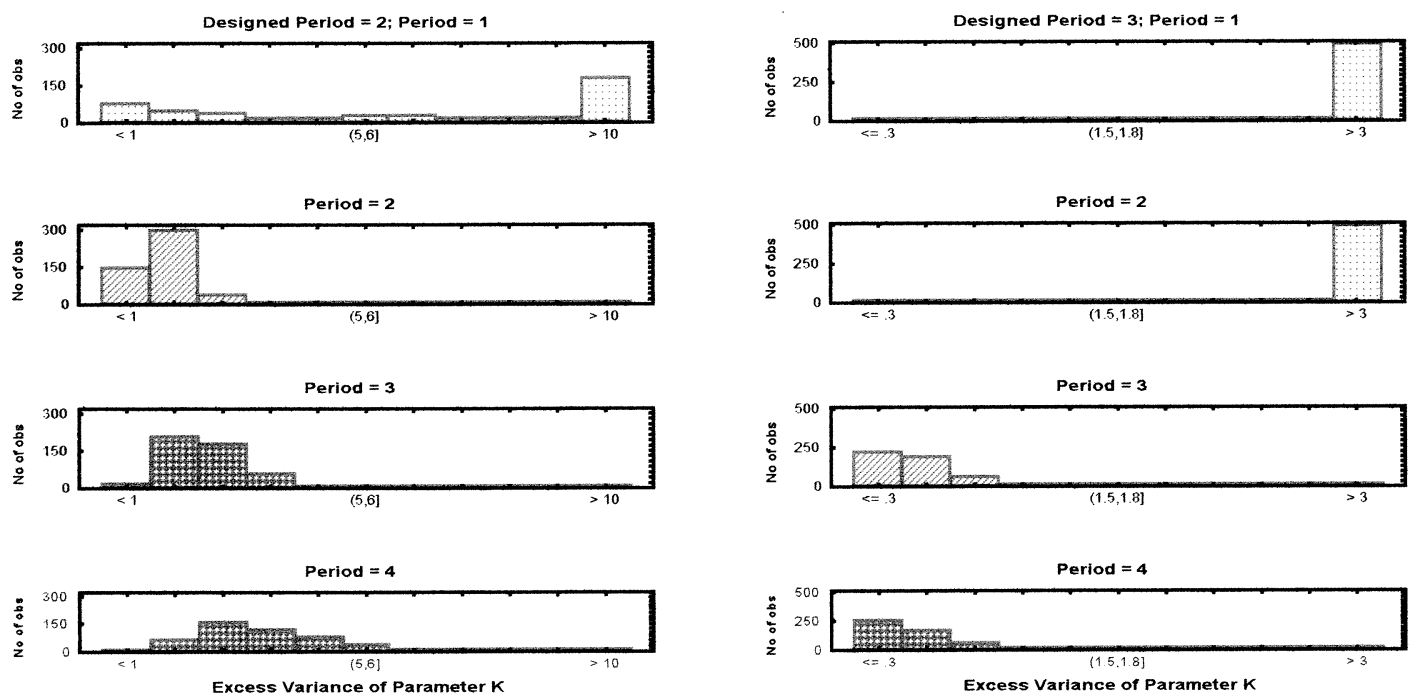

Figure 9. Relative excess variance for estimates of $K$ (under condition 1).


Figure 10. Skewness for the estimates of $K$ (under condition 1). 